\title{
Estudo do comportamento psicótico pela Análise do
} Comportamento: revisão das publicações no JEAB e JABA

\author{
Study of psychotic behavior by the Behavior Analysis: review of \\ publications in JEAB and JABA
}

\section{Estudio del comportamiento psicótico por el Análisis de Comportamiento: revisión de publicaciones em JEAB y JABA}

\author{
Fernanda Alves dos Santos' ${ }^{1}$, Karina de Lima Santos², Lívia Ferreira Godinho Aureliano ${ }^{3}$
}

[1] [2] [3] Universidade São Judas Tadeu I Título abreviado: Comportamento psicótico no JEAB e JABA I Endereço para correspondência: Fernanda A. dos Santos: Av. Ministro Petrônio Portela, 1901, bloco A, apto. 91, CEP 02802-120, Freguesia do Ó, São Paulo, SP. I Email: fernandaalves03@yahoo.com.br 
Abstract: This paper aimed to review and analyze behavior-analytic literature on psychotic behavior. Twenty-five related articles were selected on JEAB and JABA. The selected articles were fully analyzed and categorized according to the following criteria: year of publication, research objectives, target-behaviors, type of reinforcers, main results, discussions and final considerations. The majority of the studies were published on the 1970's and aimed to evaluate the effectiveness of operant principles or modify patient's behaviors. Verbal behavior and conditioned reinforcers were the most frequent target behavior and programmed consequences, respectively. The amount of behavior-analytic studies on schizophrenia and psychotic behavior published on JEAB and JABA is small if compared to other mainstream journals during the same period. Behavior Analysis should intensify research on this topic focusing on evaluating treatment effectiveness with psychotic population.

Keywords: schizophrenia, psychosis, behavior analysis.

Resumen: Este trabajo tuvo como objetivo revisar y analizar la literatura sobre la conducta psicótica basado en estudios de Análisis de la Conducta. Se seleccionaron 25 artículos relacionados en las revistas JEAB y JABA. El material fue totalmente analizado y presentado de acuerdo a su año de publicación, objetivos de investigación, objetivo-comportamientos, reforzadores utilizados, los principales resultados, discusiones y consideraciones finales. La década de 1970 es un rico período de publicación, en la mayoría de los estudios se pretende evaluar la eficacia de los principios operantes o modificar las conductas del paciente. Verbal fue el comportamiento más analizado, siendo la práctica acondicionada de reforzo la más popular. A pesar de los muchos estudios encontrados acerca de la esquizofrenia y conducta psicótica, la cantidad de investigaciones por los analistas de la conducta era pequeña en comparación con lo que se ha publicado en los últimos decenios. Por lo tanto, se cree que los analistas de la conducta deben construir nuevos conocimientos sobre el tema para que el rendimiento dentro de esta población sea más eficaz.

Palabras-clave: esquizofrenia, psicosis, conducta psicótica, análisis de la conducta. 
As síndromes psicóticas são caracterizadas pela presença de sintomas típicos, como alucinações, delírios, pensamento desorganizado e comportamentos bizarros, sendo a esquizofrenia sua principal representante (Dalgalarrondo, 2008).

As síndromes psicóticas e a esquizofrenia são diferenciadas nos manuais psiquiátricos, como por exemplo, no DSM-IV (2002), no qual delírios, alucinações acentuadas, desorganização da fala ou do comportamento, inclusive, perturbação motora, caracterizam a esquizofrenia. No mesmo manual, outros transtornos, como o transtorno psicótico devido a uma condição médica geral ou induzido por substância, são caracterizados apenas por delírios ou alucinações, enquanto que nos transtornos delirante e psicótico compartilhado, apenas o delírio é destacado como sintoma.

De acordo com a predominância dos sintomas, a esquizofrenia pode ser classificada em alguns subtipos. A Classificação Internacional de Doenças - CID 10 (OMS, 1993) a subdivide em seis: paranóide, hebefrênica, catatônica, indiferenciada, residual e simples. O Manual Diagnóstico e Estatístico de Transtornos Mentais, até sua quarta edição (DSM-IV-TR, APA, 2002), também a subdividia em cinco subtipos, mas estes foram eliminados na quinta edição (DSM-5), quando passou-se a considerar a gravidade dos sintomas para diferenciação (APA, 2013).

Bueno e Britto (2011) afirmam que o termo "psicótico" já recebeu diversas denominações, mas nenhuma foi amplamente aceita, sendo sua definição limitada à existência de delírios e alucinações. $\mathrm{Na}$ esquizofrenia, além desses sintomas, verifica-se também uma desorganização do pensamento, sendo a doença caracterizada como um comportamento amplamente desorganizado.

Estima-se que a prevalência da esquizofrenia na população mundial seja de aproximadamente $1 \%$. No Brasil, ocupa 30\% dos leitos psiquiátricos hospitalares e está em segundo lugar dentre os motivos para as primeiras consultas psiquiátricas ambulatoriais. Embora a doença acometa ambos os sexos, o risco dos homens a desenvolverem é de 1,4 a 2,3 vezes maior do que entre as mulheres, sendo a idade média de incidência nos homens entre 15 e 25 anos, e nas mulheres entre os 25 e 35 anos (Lara, Gama \& Abreu, 2004; Zanetti \& Elkis, 2008).
A esquizofrenia pode ter diversas apresentações, uma vez que a expressão dos sintomas varia de acordo com cada paciente e com o passar do tempo (Louzã Neto, Azevedo \& Macedo, 2007). Os sintomas podem ser agrupados em três categorias: (1) positivos (ou psicóticos), que são características apresentadas pelos pacientes que estão ausentes em pessoas saudáveis, como delírios e alucinações; (2) negativos, que são características normalmente presentes em indivíduos saudáveis que estão diminuídos ou ausentes nos pacientes, como embotamento afetivo, avolição (falta de vontade), anedonia (falta de prazer) e alogia (discurso empobrecido); e (3) de desorganização, como comportamentos bizarros, afetos inapropriados, desorganização do pensamento e distúrbios de atenção (Lara et al., 2004; Zanetti \& Elkis, 2008).

De acordo com Martone e Zamignani (2002), os manuais psiquiátricos, CID 10 (1993) e DSM-IV (2002), auxiliam o início da avaliação, permitem padronização e troca de informação entre profissionais, além de guiarem pesquisas. As descrições apresentadas colaboram para uma predição comportamental e possibilitam a elaboração de estratégias iniciais de tratamento. No entanto, os autores ressaltam os limites dessas descrições, uma vez que seu caráter é estritamente topográfico e baseado em critérios estatísticos de "normalidade". Britto (2005) acrescenta que "nas descrições topográficas dos manuais diagnósti$\cos [. .$.$] o comportamento verbal bizarro foi psicopa-$ tologizado como sintoma da esquizofrenia" (p. 40).

Embora a utilização dos manuais apresente vantagens, sua visão baseada no modelo médico tende a provocar rejeição por parte dos analistas do comportamento (Banaco, Zamignani \& Meyer, 2010). Além disso, Pérez-Alvárez (2004) ressalta que os sistemas classificatórios são utilizados não só para o desenvolvimento do conhecimento científico, mas para a "legitimização da psiquiatria como uma especialidade médica” (p. 173).

No mesmo sentido, Maciel e Modesto (2008) afirmam que a utilização dos manuais psiquiátricos é necessária para que haja efetividade na comunicação entre os mais variados profissionais. Todavia, as autoras ressaltam que os analistas do comportamento devem considerar aspectos que estão além da descrição sintomática para que quaisquer intervenções clínicas sejam efetivas. 
É necessário, portanto, que o clínico busque as variáveis envolvidas nos comportamentos denominados "patológicos", pois os rótulos dados a tais comportamentos criam uma ilusão de explicação que é circular e não se mantêm em uma análise mais rigorosa (Hunziker \& Samelo, 2011): dizer que o sujeito é esquizofrênico porque tem alucinações convive com a explicação de que ele tem alucinações porque é esquizofrênico.

Cabe salientar as diferenças entre os diversos modelos de psicopatologia existentes: o médico, o quase-médico e o analítico-comportamental. No modelo médico, doenças subjacentes seriam expressas pelo aparecimento de transtornos, os quais receberiam, geralmente, tratamento farmacológico. O modelo quase-médico, classificação dada à psicologia clínica que atribui o desenvolvimento de transtornos a causas intrapsíquicas, diferencia-se do anterior apenas por considerar que a utilização de fármacos não é essencial para o tratamento das doenças. Ambos os modelos baseiam-se nos critérios estatísticos de normalidade, ou seja, os comportamentos que diferem da maioria são considerados anormais (Banaco et al., 2010).

Por outro lado, o modelo analítico-comportamental defende uma análise funcional dos comportamentos classificados como psicopatológicos, considerando as contingências que os estabeleceram e os mantêm. Nesse sentido, muitas vezes observa-se a influência de contingências de punição, fuga e esquiva, que acarretam em dificuldades no desenvolvimento de atividades cotidianas e, consequentemente, em prejuízos pessoais e sociais (Banaco et al. 2010; Sidman, 1989/2003; Vilas Boas, Banaco \& Borges, 2011).

Sidman (1989/2003) ressalta que a definição do termo "anormalidade", adotada nos modelos anteriores, não envolve análises de processos comportamentais, mas "grosseiros critérios estatísticos", nos quais psiquiatras e psicólogos baseiam-se para tentar "curar qualquer ação que se desvie do usual" (p.193). O autor ainda destaca que tal definição é contraditória, uma vez que "vivemos em uma sociedade complexa" (p. 194), na qual alguns comportamentos são aceitos em determinadas comunidades e em outras, não.

Vilas Boas, Banaco e Borges (2011) acrescentam que o que determina a classificação de pa- drões comportamentais como transtornos mentais são os padrões socialmente aceitos estabelecidos pelas práticas culturais, e os que violam tais padrões são considerados como "anormais" ou "psicopatológicos".

Bueno e Britto (2011) afirmam que o indivíduo esquizofrênico é influenciado por contingências passadas e atuais, interagindo "tanto com as contingências ambientais de reforçamento e punição quanto com os efeitos de sua história" e que os comportamentos ditos psicóticos (delirar e alucinar) "são comportamentos verbais controlados pelas consequências verbais e não verbais que produzem" (p. 6). Pode-se dizer, então, que o que a literatura psiquiátrica define como sintoma psicótico, na análise do comportamento pode ser compreendido como comportamento e, portanto, deve ser analisado como tal (Martone \& Zamignani, 2002), considerando-se a interação entre os níveis de seleção e as contingências que o mantêm.

Skinner (1956/1979) sintetiza, afirmando que "o comportamento do psicótico é simplesmente parte e parcela do comportamento humano" (p. 189) e, "como todo e qualquer comportamento, constitui parte do mundo de eventos observáveis, ao qual se aplicam os métodos poderosos da ciência natural" (p. 196).

\section{Comportamento Psicótico como Objeto de Pesquisa}

A extensão dos princípios operantes para sujeitos humanos iniciou-se quando B.F. Skinner e seu orientando, Ogden Lindsley, com o auxílio de Harry Solomon, então chefe do Departamento de Psiquiatria e diretor do Boston Psychopathic Hospital, fundaram, em 1953, o Harvard Medical School Behavior Research Laboratory (BRL), no Metropolitan State Hospital em Waltham, Massachusetts. No laboratório, foram montadas duas salas experimentais à prova de som, facilmente limpas e difíceis de serem destruídas; eram equipadas com uma máquina fornecedora de reforços, que continha um puxador (denominado manipulandum) feito de ferro e latão, o qual poderia ser puxado até 10.000 vezes por hora (Lundin, 1969/1977; Rutherford, 2003). 
O objetivo do laboratório não era tentar modificar ou modelar o comportamento dos participantes com fins terapêuticos, mas verificar se o paradigma operante e o método experimental eram aplicáveis a humanos, tal qual a sujeitos infra-humanos; e, neste caso, se o paradigma seria um método apropriado para investigar as propriedades funcionais do comportamento de psicóticos (Martone \& Zamignani, 2002; Rutherford, 2003).

Em 1954, Skinner, Solomon e Lindsley apresentaram a pesquisa inicial do laboratório, que contou com 15 pacientes do sexo masculino, com idade média de 38 anos e tempo médio de hospitalização de 17 anos. Nesse experimento, os participantes eram apresentados a uma variedade de potenciais reforçadores (doces, cigarros, moedas ou fotos de garotas pin-ups) e solicitados a escolher qual era o seu preferido. O participante era levado para a sala experimental onde era instruído a puxar a alavanca (manipulandum) ou deixado livre para explorar a sala até que a resposta de puxar a alavanca ocorresse. Foram realizados dois esquemas de reforçamento positivo: intervalo variável de 1 minuto (VI 1) e razão fixa de 20 respostas (FR 20). Os pesquisadores concluíram que o efeito dos diferentes esquemas de reforçamento no comportamento dos participantes foi similar ao apresentado por ratos, pombos e cachorros, e recomendaram mais estudos, inclusive sobre o efeito de drogas no comportamento operante (Rutherford, 2003).

O laboratório funcionou até 1965, mas Skinner deixou de ter envolvimento direto com ele no final dos anos 50, período no qual Lindsley já havia obtido diploma de $\mathrm{PhD}$ e dedicava-se em tempo integral às pesquisas. Alguns resultados comuns foram observados na análise de cerca de 50 registros de pesquisas com pacientes crônicos (esquizofrênicos e pacientes com distúrbios orgânicos e funcionais) realizadas durante o funcionamento do laboratório, tais como irregularidade na frequência das respostas e nas pausas realizadas nos esquemas de reforçamento (maiores no esquema de FR do que no de VI, quando em sujeitos "normais" verifica-se o inverso) e grande resistência à extinção. Tais resultados permitem dizer que há uma alteração importante no padrão de responder dos pacientes caracterizados como psicóticos em relação ao responder típico de humanos, mas, até hoje, as expli- cações para essas diferenças são pouco conclusivas (Lundin, 1969/1977; Martone \& Zamignani, 2002; Rutherford, 2003).

A esquizofrenia como problema comportamental, então, passou a ser estudada nas décadas de 50 e 60, utilizando a aplicação de princípios do condicionamento operante, através da manipulação de variáveis de reforçamento (positivo e negativo), extinção e saciação (Britto, 2005; Martone \& Zamignani, 2002; Silva, 2006).

Dentre as técnicas utilizadas, destaca-se o programa de economia de fichas (Token Economy), desenvolvido por Ayllon e Azrin (1965). Nesse sistema, fichas eram dispensadas contingentes a emissão de comportamentos selecionados e, posteriormente, poderiam ser trocadas por variados itens. De acordo com Kazdin (1982), o programa foi estendido para outros ambientes e outras populações, sendo aplicado tanto para pacientes psiquiátricos crônicos quanto para pacientes com doenças agudas, síndromes cerebrais orgânicas, doenças psicossomáticas e crianças autistas, além de novas áreas de pesquisa como medicina comportamental, ecologia comportamental, psicologia comunitária e psicologia geriátrica.

No Brasil, publicações de pesquisa aplicada sobre o tema são encontradas ${ }^{1}$ a partir do início dos anos 2000 e, excetuando-se alguns trabalhos isolados (Bandeira, Machado \& Pereira, 2002; Martone, 2005), grande parte dos trabalhos foram realizados por Britto e colaboradores (Britto, Rodrigues, Alves \& Quinta, 2010; Britto, Rodrigues, Santos \& Ribeiro, 2006; Epaminondas, 2010; Felipe, 2009; Marcon, 2010; Miranda, 2005; Santana, 2008; Santos, 2007; Silva, 2005).

Encontram-se, ainda, na literatura internacional, pesquisas que utilizam a Terapia de Aceitação e Compromisso (ACT) para tratamento dos sintomas psicóticos positivos, especialmente as alucinações (Bach \& Hayes, 2002; Carmona, Cangas, GarcíaMontes \& Pedro, 2009; García-Montes \& PérezÁlvarez, 2005; García-Montes, Soriano, López \& Basurto, 2004).

Diante da relevância social do tema, destaca-se a necessidade de verificar o que foi produzido pela Análise do Comportamento acerca do com-

1 Disponibilizadas na internet. 
portamento psicótico, a fim de orientar e ampliar o campo de atuação e pesquisa dos analistas do comportamento. Scotti, McMorrow e Trawitzki (1993), Martone e Zamignani (2002) e Camargo (2008), fizeram revisões sobre o tema. O trabalho de Scotti et al. (1993) visava analisar publicações que enfocassem a aplicação da terapia comportamental a desordens psiquiátricas crônicas, no período de 1963 a 1988 (Camargo, 2008). O trabalho de Camargo (2008) objetivou continuar tal análise, cobrindo o período de 1988 a 2007, mas considerando apenas pesquisas com intervenções comportamentais ${ }^{2}$ aplicadas à esquizofrenia. Já Martone e Zamignani (2002), realizaram um levantamento parcial da literatura behaviorista radical sobre esquizofrenia, considerando publicações a partir da década de 60 .

O objetivo geral deste estudo foi realizar um levantamento de pesquisas publicadas em dois periódicos internacionais, JEAB e JABA, desde as primeiras edições, contemplando o período de 1959 a 2011. O trabalho atual difere-se dos anteriores por cobrir um período ainda não analisado (2007 a 2011) e por considerar apenas periódicos especializados em Análise do Comportamento, diferentemente do estudo de Scotti et al. (1993), que utilizou jornais e revistas representativas e reconhecidas nas áreas da psiquiatria, psicologia e terapia comportamental, e de Carmago (2008), que utilizou a maior parte dos jornais selecionados por Scotti et al. (1993) acrescendo também periódicos nacionais. Destaca-se ainda, que a presente pesquisa teve como objetivo analisar o que tem sido produzido pela comunidade de analistas do comportamentos sobre o comportamento psicótico, enquanto que estudos anteriores objetivaram verificar especificamente a utilização da terapia/ intervenção comportamental em pacientes diagnosticados como psicóticos.

Analisou-se especificamente o ano de publicação, objetivos das pesquisas, comportamentos-alvo, reforçadores utilizados, principais resultados, discussão e considerações finais dos artigos.

2 Fizeram parte da análise "artigos que descreviam uma técnica comportamental, mas que não necessariamente adotavam uma postura de terapia comportamental" (Camargo, 2008, pg. 23).

\section{Método}

\section{Material}

Como material foram utilizados artigos que abordassem o comportamento psicótico sob a perspectiva da Análise do Comportamento, arrolados em dois jornais especializados em Análise do Comportamento, o JEAB e o JABA, e disponibilizados através do site: seab.envmed.rochester.edu.

O Journal of the Experimental Analysis of Behavior (JEAB) e o Journal of Applied Behavior Analysis (JABA) foram escolhidos como fontes de pesquisa porque ambos são referência na publicação de pesquisas em Análise do Comportamento, sendo o enfoque principal do JEAB artigos experimentais relevantes para a compreensão do comportamento dos organismos, enquanto que no JABA as pesquisas publicadas abordam a aplicação da Análise do Comportamento a problemas de importância social.

\section{Procedimento}

Para obtenção dos artigos, acessou-se o site seab. envmed.rochester.edu. Selecionou-se, então, a seção "JABA Abstracts" (resumos do JABA), que está localizada no topo da página. Já nessa seção, no campo "search for" (procurar) foram digitadas, individualmente, cada uma das palavras-chaves: "Psychiatric" (psiquiátrico), "Psychiatry" (psiquiatria), "Psychoses" (psicoses), "Psychosis" (psicose), "Psychotic" (psicótico), "Schizophrenia" (esquizofrenia) e "Schizophrenic" (esquizofrênico); no campo "match" (corresponder), manteve-se a seleção em "any search words" (qualquer palavra da pesquisa), e no "Journal" (jornal) selecionou-se "both" (ambos), para que a pesquisa fosse realizada tanto no JABA quanto no JEAB. Em seguida, clicou-se em "submit" (submeter) para que a pesquisa fosse realizada.

No total, retornaram 72 artigos. Eliminando-se os artigos repetidos resultantes de pesquisas com palavras-chave diferentes, restaram 53. Através de uma análise inicial dos resumos, verificou-se que 23 não tinham como objetivo estudar o comportamento psicótico ou não faziam referência a pacientes com tais comportamentos. 
Restaram 30 artigos. Destes, cinco foram excluídos, visto que dois só continham resumo, os quais foram insuficientes para preencher os dados da tabulação, e em três outros casos, a caracterização dos comportamentos psicóticos não estava de acordo com a estabelecida pela literatura pesquisada, ou seja, os comportamentos denominados psicóticos não envolviam delírios, alucinações ou desorganização. Os 25 artigos restantes (vide Anexo A) foram tabulados utilizando o programa Microsoft Excel. Inicialmente, foram transcritos os objetivos, participantes, procedimentos e resultados/discussão/ considerações finais dos artigos. Posteriormente, cada transcrição foi subdividida e categorizada de acordo com os objetivos específicos. Os resultados estão apresentados a seguir.

\section{Resultados e Discussão}

A Figura 1 apresenta a divisão dos artigos conforme o ano de publicação. Os primeiros artigos datam de 1959 (2 artigos) e foram agrupados com os escritos na década de 1960, bem como o artigo publicado em 2011 foi agrupado com os publicados na década de 2000.

Observa-se que a maior parte dos trabalhos (12) foi publicada na década de 70, sendo 1973 e
1979 os anos com mais trabalhos publicados (três cada um); em seguida, está a década de 60, com sete publicações. A partir da década de 1980, a produção nessa área decresceu consideravelmente, diminuindo de 12 trabalhos na década anterior, para apenas uma publicação, datada de 1987. As décadas seguintes também apresentaram pequena quantidade de trabalhos publicados, sendo dois na década de 1990 e três na de 2000 .

Tal resultado corrobora os achados nos trabalhos de Martone e Zamignani (2002), que apontam diminuição de trabalhos a partir da década de 1980, e de Camargo (2008), que indica uma estabilização no número de publicações a partir de 1988, com uma média de 1,05 artigo por ano.

De acordo com Martone e Zamignani (2002), a diminuição das publicações a partir da década de 1980 deveu-se, principalmente, às críticas aos métodos utilizados nestas pesquisas, feitas tanto fora quanto dentro da comunidade de analistas do comportamento. Como consequência, houve uma diminuição na utilização dos procedimentos comportamentais e a retirada dos programas das instituições públicas e privadas. Ao invés de gerarem a busca por novos métodos de tratamento aos pacientes psicóticos, as críticas parecem ter levado a um abandono das pesquisas e do desenvolvimento de tecnologias para lidar com o transtorno por parte dos analistas do comportamento.

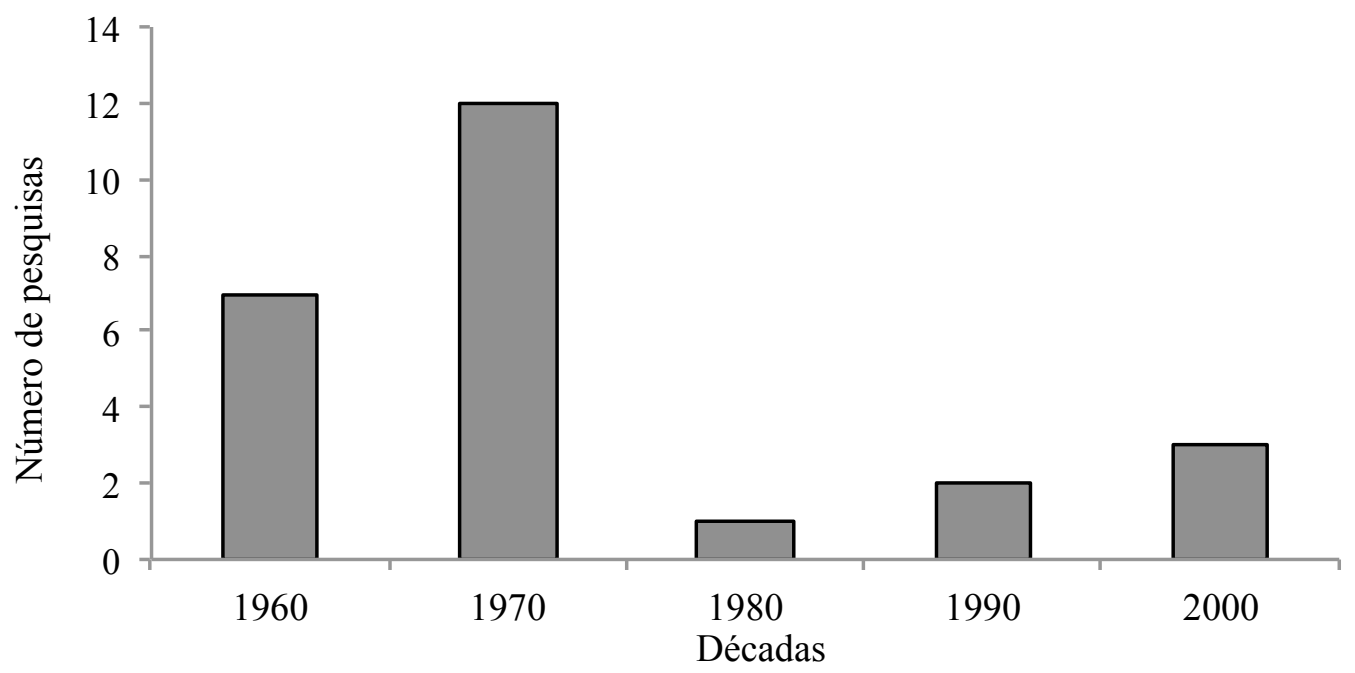

Figura 1. Quantidade de artigos publicados por década 
A falta de desenvolvimento de novas tecnologias também pode ser verificada pela análise dos objetivos dessas publicações (Figura 2).

Observa-se que na década de 60, quatro das sete pesquisas do período intencionavam avaliar a efetividade de princípios operantes. Dentre essas pesquisas, cabe destacar a realizada por Ayllon e Azrin, em 1965, The measurement and reinforcement of behavior of psychotics, pioneira na utilização de fichas como reforçadores condicionados em instituições psiquiátricas e que levou ao desenvolvimento da Token Economy (programa de economia de fichas - PEF).

$\mathrm{Na}$ década de 70 , algumas pesquisas continuaram avaliando a efetividade dos princípios operantes na população denominada psicótica (quatro pesquisas), mas já observa-se um aumento nos trabalhos cujo objetivo era a modificação do comportamento destes pacientes, sendo este o foco de cinco pesquisas.

A publicação da década de 80 teve como objetivo pesquisar a efetividade dos princípios operantes, mas nas décadas seguintes o foco passou a ser a modificação do comportamento, sendo este o objetivo de uma pesquisa na década de 90 e de duas na década de 2000 .
É possível verificar que, apesar das primeiras pesquisas já comprovarem a efetividade de princípios operantes em pacientes esquizofrênicos, estes continuaram sendo objeto de estudo, em detrimento de pesquisas que focassem a alteração do comportamento destes pacientes.

Apesar disso, as tentativas de modificar o comportamento da população psiquiátrica através do desenvolvimento de procedimentos efetivos marcaram o início da prática do analista do comportamento e diferenciou a abordagem analítico-comportamental das demais abordagens psicológicas da época, que entendiam tais comportamentos como refratários e, portanto, sem solução (Guedes, 1993; Martone \& Zamignani, 2002).

Guedes (1993) destaca que os primeiros trabalhos de modificação do comportamento foram realizados com pacientes muito graves e que tal gravidade, muitas vezes, dificultava inclusive as relações interpessoais.

A participação de pacientes internados em instituições psiquiátricas nas pesquisas foi alvo de críticas pela comunidade científica, uma vez que tais instituições eram ambientes controlados que possibilitavam a criação de contingências arbitrárias para a alteração do comportamento. Tal con-

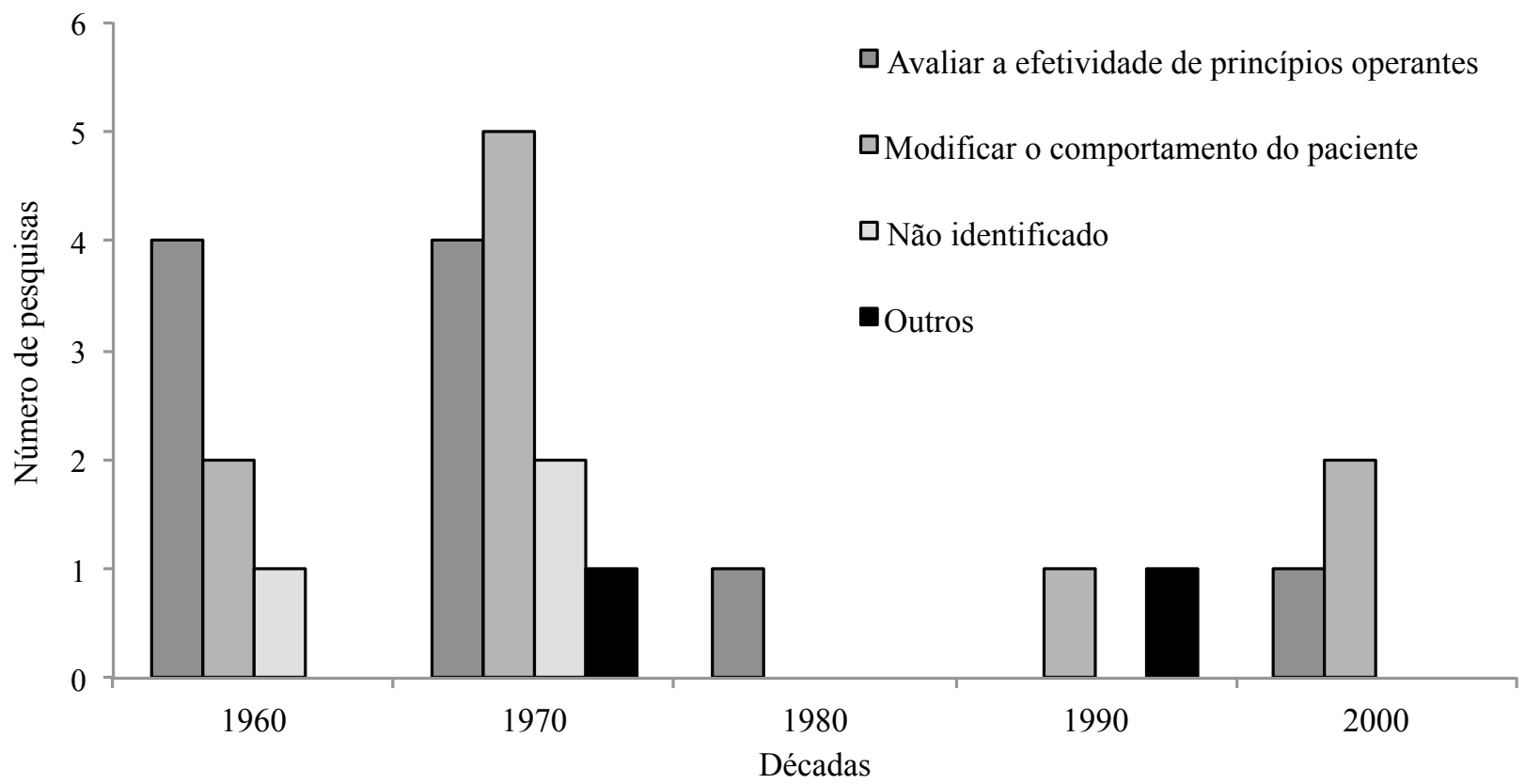

Figura 2. Distribuição dos artigos, por década, de acordo com os objetivos descritos nos artigos. 
trole foi considerado uma ofensa à liberdade pessoal (Guedes, 1993; Holland, 1978; Martone, 2005; Martone \& Zamignani, 2002;).

Além disso, alegava-se que os comportamentos modificados nesses ambientes não se manteriam fora das instituições, pois uma vez que o comportamento é mantido pelas contingências, mudanças duradouras dependem da alteração de contingências que produziram e mantêm o comportamento original, não sendo suficiente a criação de contingências artificiais para modificá-lo (Guedes, 1993; Holland, 1978; Martone, 2005; Martone \& Zamignani, 2002). Nesse sentido, pode-se dizer que houve "má compreensão da própria proposta teórica que sustentava essa prática" (Guedes, 1993, p. 82).

Outra crítica feita era que as técnicas empregadas visavam à modificação de respostas discretas e que tais mudanças eram superficiais e irrelevantes (Guedes, 1993; Martone \& Zamignani, 2002). Os comportamentos selecionados como alvo de intervenção nas pesquisas analisadas foram classificados em 08 categorias: comportamento verbal, alimentação, autocuidado, habilidades sociais, comportamentos agressivos, atividades domésticas, outros excessos comportamentais, outros.

Algumas pesquisas foram incluídas em mais de uma categoria e a distribuição pode ser verificada na Figura 3.

Verifica-se que na década de 60 , os comportamentos envolvidos com a alimentação foram o principal foco de intervenção. O comportamento verbal que foi alvo de intervenção em duas pesquisas, passa a ser o principal comportamento estudado nas décadas seguintes, presente em sete publicações na década de 70 e em uma da década de 80 . $\mathrm{Na}$ década de 90 prevalece o estudo de habilidades sociais, já na década de 2000 o foco de intervenção volta a ser o comportamento verbal.

Observa-se que, inicialmente, os comportamentos alvo de intervenção não são os comportamentos típicos de esquizofrênicos, como os sintomas positivos ou negativos, mas, como destacado por Ayllon e Michael (1959), na pesquisa The psychiatric nurse

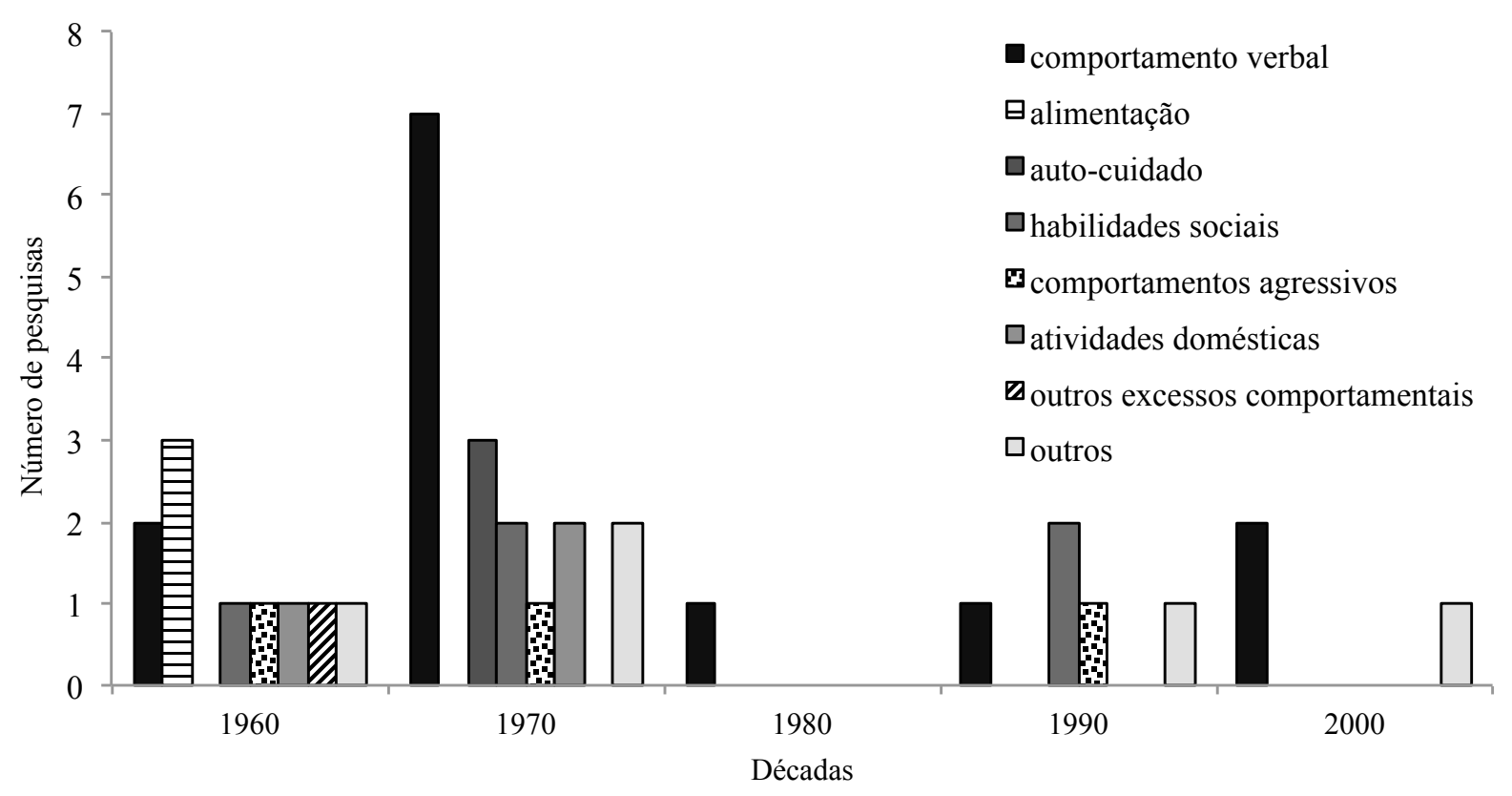

Figura 3. Distribuição dos artigos, por década, de acordo com o comportamento-alvo estudado. 
as a behavioral engineer, aqueles que são emitidos em alta frequência e cuja persistência permitia várias tentativas de alterá-los. Como já discutido anteriormente, tal escolha pode estar relacionada com o fato de que as primeiras pesquisas objetivavam apenas a verificação da efetividade de princípios operantes nessa população e não o seu tratamento.

A partir da década de 70, o foco passa a ser o comportamento verbal dos pacientes e a intervenção visava a diminuição de falas psicóticas/ inapropriadas e aumento das falas não psicóticas/ apropriadas. Nesse período ainda, verifica-se a utilização do treino de habilidades sociais nas pesquisas buscando a melhora do desempenho social destes pacientes, principalmente em respostas relacionadas com o comportamento verbal, como uso apropriado das mãos e contato visual adequado.

Apesar de os estudos passarem a enfocar o comportamento verbal, Layng e Andronis (1984) destacam que nessas pesquisas tais respostas eram vistas "simplesmente como topografias a serem eliminadas" (p. 139), ao invés de serem analisadas funcionalmente, como operantes verbais skinnerianos. Os autores defendem que, como comportamentos operantes, os padrões delirantes e alucinatórios dos pacientes são mantidos por suas consequências. No entanto, tais comportamentos são, comumente, classificados como alvo de intervenção clínica pelos custos que trazem para o indivíduo (como perda de emprego, internação, estigmatização social), mas não são feitas referências aos possíveis reforçadores que os mantêm.

Além disso, embora algumas pesquisas realizassem uma análise de contingências, esta era restrita a algumas respostas e o objetivo era a classificação destas como adaptativas ou não adaptativas. A intervenção, então, objetivava a eliminação das respostas não adaptativas (Martone \& Zamignani, 2002).

Em pesquisas mais recentes, como as realizadas por Dixon, Benedict e Larson (2001) e por Wilder, Masuda, O'Connor e Baham (2001), verifica-se a replicação do modelo de análise funcional experimental desenvolvido por Iwata et al., em 1982, que objetiva estudar as condições antecedentes e consequentes do comportamento, através da utilização de diferentes condições experimentais. Tal modelo tem sido utilizado em pesquisas aplicadas na análise do comportamento com crianças e adolescentes que apresentam comportamento autolesivo, bem como com outras populações, como os pacientes psiquiátricos (Iwata, Dorsey, Slifer, Bauman \& Richman, 1982/1994; Marcon, 2010; Martone, 2005).

A extensão do modelo de Iwata et al. (1994) mostra a preocupação dos analistas do comportamento com as causas do comportamento "problema", possibilitando intervenções mais efetivas, a despeito das críticas realizadas em relação à alteração do comportamento utilizando contingências arbitrárias (Marcon, 2010). No entanto, a passagem dos procedimentos de modificação comportamental para procedimentos envolvendo análise funcional, fez com que os terapeutas comportamentais deixassem para trás "a solução de problemas concretos, a rapidez da terapia, os registros [e] a confiabilidade na relação procedimento/resultados" (Guedes, 1993, p. 82), adotando uma prática restrita a consultórios, o que talvez ajude a explicar a diminuição de pesquisas sobre o assunto.

Observa-se que em algumas pesquisas, por exemplo, Reinforcement and instructions with mental patients, de Ayllon e Azrin (1964), a seleção da resposta ou da variável a ser modificada (introduzida ou retirada) era alterada em função dos resultados de um primeiro experimento. Uma vez que os resultados da primeira intervenção indicaram que instruções associadas à contingência de reforçamento positivo eram mais efetivas para controlar a resposta de pegar talheres das pacientes do que apenas a contingência de reforçamento positivo, uma segunda intervenção foi realizada. Assim, para verificar se o comportamento estava apenas sob controle das instruções, estas eram apresentadas isoladas e depois associadas à contingência de reforçamento positivo, utilizando-se reforçadores primários e generalizados.

A distribuição dos reforçadores utilizados nas demais publicações pode ser verificada na Figura 4. Algumas pesquisas utilizaram mais de um tipo de reforçador, sendo incluídas em mais de uma categoria.

Verifica-se que na década de 60 os principais reforçadores utilizados foram os generalizados e os condicionados, presentes em três pesquisas cada. $\mathrm{Na}$ década de 70, os reforçadores condicionados foram os mais utilizados (seis pesquisas), seguidos pelos reforçadores sociais (três pesquisas). $\mathrm{Na}$ 


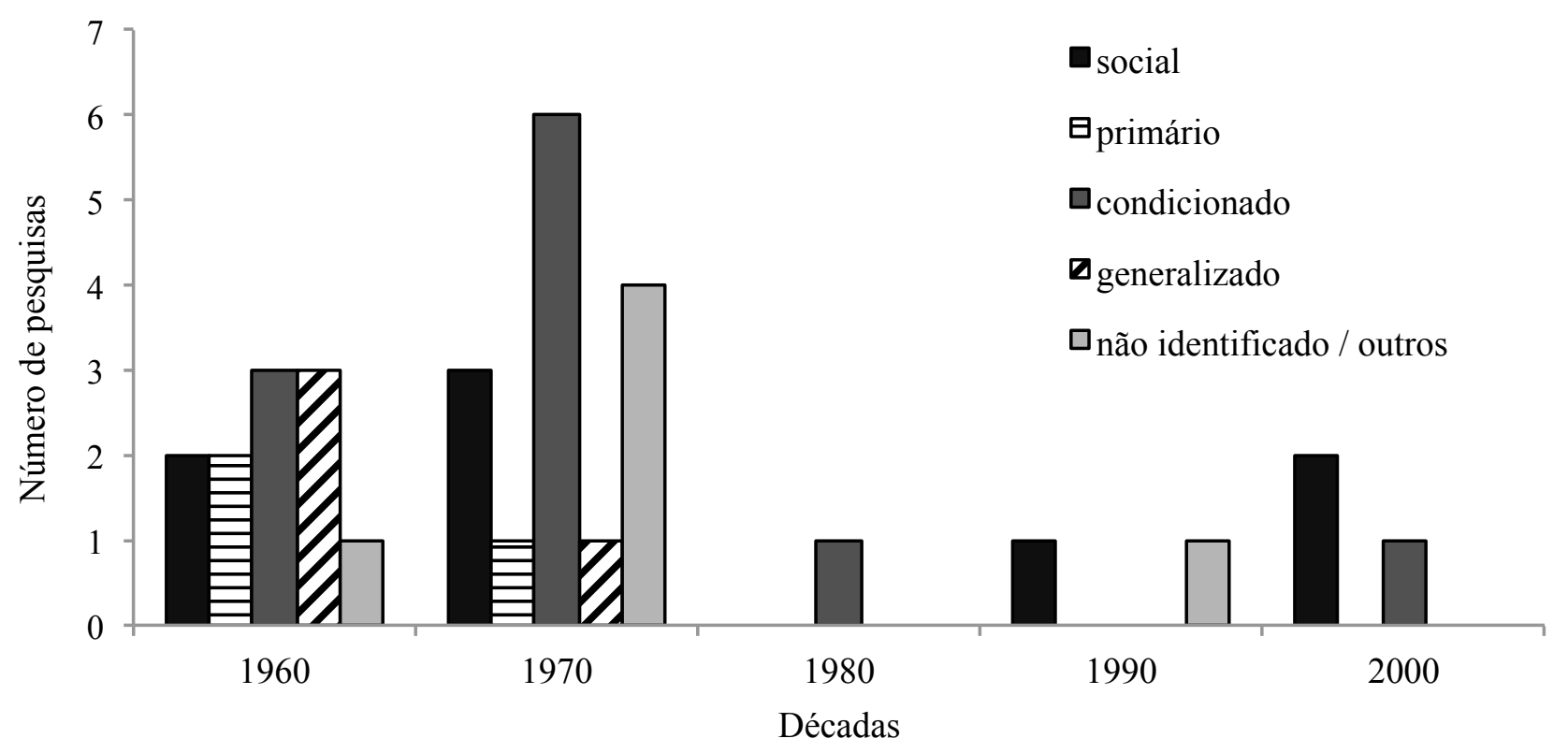

Figura 4. Distribuição dos artigos, por década, de acordo com o tipo de reforçador utilizado.

pesquisa da década de 80 , foram utilizados apenas reforçadores condicionados, uma da década de 90 utilizou reforçadores condicionados e sociais e a outra não foi possível identificar. Na década de 2000, duas pesquisas utilizaram reforçadores sociais e uma utilizou reforçadores condicionados.

As pesquisas cujas contingências foram de reforçamento negativo utilizaram como estímulo aversivo derrubar comida na roupa da paciente enquanto a estivesse alimentando (Ayllon \& Michael, 1959), retirada da atividade solicitada (Mitchell \& Stoffelmayr, 1973) e solicitações do comportamento desejado repetidas por um membro da equipe (Fichter, Wallace, Liberman \& Davis, 1976).

A alta utilização de reforçadores condicionados foi determinada pelo uso do sistema de economia de fichas, que foi bastante difundido a partir da década de 60. Conforme especificado por Ayllon e Azrin (1965), na pesquisa The measurement and reinforcement of behavior of psychotics, o principal benefício da utilização das fichas é suprir o atraso entre a resposta e o reforçador na contingência, além de eliminar a necessidade de descobrir qual reforçador é eficiente para cada indivíduo, uma vez que cada um pode expressar suas preferências individuais na troca das fichas. Kazdin e Bootzin (1972) acrescentam que os reforçadores condicionados permitem o reforçamento da resposta em qualquer momento, podem ser usados para manter o desempenho por um longo período de tempo, permitem que sequências de respostas sejam reforçadas sem interrupções, mantêm sua propriedade reforçadora devido à independência de estados de privação e são menos sujeitos aos efeitos da saciação.

O sistema, no entanto, recebeu diversas críticas a despeito de sua aparente funcionalidade. A dificuldade em treinar a equipe para aplicação do programa, aumentar a capacidade do cliente de responder às contingências e superar a resistência deste ao programa eram alguns dos obstáculos enfrentados para a aplicação da economia de fichas. Outra crítica realizada era de que contingências arbitrárias não seriam eficientes na alteração dos comportamentos e que havia uma discrepância entre os reforçadores dispensados e as contingências naturais do comportamento dos pacientes, sendo a generalização fruto de outras variáveis (Álvarez, 1996, citado por Martone \& Zamignani, 2002; Kazdin, 1982; Kazdin \& Bootzin, 1972; Martone \& Zamignani, 2002).

Nesse sentido, cabe destacar que em um dos experimentos realizado por Ayllon e Azrin (1965), foi considerada a possibilidade de reforçadores so- 
ciais estarem mantendo a resposta dos participantes ao invés ou conjuntamente com os reforçadores condicionados.

Verificou-se que, no geral, a maioria dos estudos atingiu os objetivos traçados, comprovando a efetividade de esquemas operantes e reforçadores diversos na modificação do comportamento de paciente psicóticos.

Além disso, Stewart, Van Houten e Van Houten (1992) atingiram parcialmente o objetivo geral do experimento (modificar o comportamento do paciente), pois observaram o aumento das interações sociais dos participantes apenas com seus respectivos terapeutas, não havendo a generalização do comportamento social com outras pessoas.

No que se refere às considerações das pesquisas, Hersen e Bellack (1976), em A multiple-baseline analysis of social-skills training in chronic schizophrenics, ainda que tenham observado a efetividade do reforço social e dos treinos de habilidades sociais em indivíduos com esquizofrenia, sugerem que futuros estudos sejam realizados para analisar a amplitude e a estabilidade da generalização destes comportamentos, uma vez que o reforço social pode não ser suficiente para todos os pacientes crônicos.

Ayllon e Michael (1959), sinalizaram em The psychiatric nurse as a behavioral engineer as dificuldades na utilização das enfermeiras como assistentes experimentais. De acordo com os autores, estas falhavam em seguir as instruções e eram encontrados erros sistemáticos nas observações, principalmente de classes de respostas facilmente identificáveis e de alterações sutis no comportamento. Além disso, quando as enfermeiras atribuíam o problema do paciente à idade ou ao diagnóstico deste, pouco ou nenhum esforço era realizado para descobrir e manipular variáveis ambientais relevantes. Os autores destacam ainda, que as enfermeiras não ficariam satisfeitas com nenhuma melhora menor que a cura e que, portanto, após a modificação de um comportamento dito problema, este era esquecido e outro era colocado como mais importante.

Outra objeção frequente das enfermeiras ao programa era de que as mudanças eram temporárias. No entanto, Ayllon e Michael (1959) ressaltam que a eliminação permanente dos problemas de comportamento da ala exige eliminação perma- nente das variáveis ambientais que os modelaram e os mantêm. Nesse sentido, os autores verificaram que as profissionais de enfermagem mantinham os comportamentos indesejados através da atenção ou da aprovação social destinada aos pacientes. Winkler (1970) também verificou na pesquisa Management of chronic psychiatric patients by a token reinforcement system que o comportamento passivo das pacientes, aparentemente, era reforçado pela atenção ou pelo tratamento dados a elas, podendo-se concluir, então, que os pacientes psicóticos são sensíveis às contingências ambientais.

Ayllon e Azrin, em 1965, corroboram com essa conclusão ao afirmarem que o tempo de hospitalização pode extinguir comportamentos dos pacientes e que melhores resultados com as intervenções seriam conseguidos se fossem realizadas no início da hospitalização, quando os padrões comportamentais estivessem intactos. Winkler (1970) verificou que a utilização do programa de fichas melhorou o comportamento global das pacientes, inclusive em respostas não reforçadas diretamente pelo sistema. De acordo com o relato das enfermeiras, as pacientes estavam menos apáticas e faziam mais perguntas sobre as tarefas solicitadas.

Em contrapartida, algumas pesquisas apontam para a restrição dos resultados obtidos, como na pesquisa de Wincze, Leitenberg e Agras (1972) The effects of token reinforcement and feedback on the delusional verbal behavior of chronic paranoid, que verificou que os efeitos dos procedimentos utilizados (feedback e reforçamento com fichas) foram específicos nos ambientes onde foram aplicados, com pequena generalização para outros ambientes. Patterson e Teigen (1973) também apontam que melhores resultados na generalização seriam obtidos se intervenções fossem feitas com uma variedade de locais e de pessoas.

Apenas uma pesquisa apresentou como resultado que os pacientes psicóticos respondem de forma diferente em relação aos não psicóticos. Operant behavior in psychiatric patients (Bulock, 1959) verificou que o controle discriminativo em pacientes psicóticos crônicos é menor quando comparado com não psicóticos e, até mesmo, com psicóticos agudos. Infelizmente, como só foi possível o acesso ao resumo dessa pesquisa, não foram encontradas mais informações sobre tal diferença. 


\section{Conclusão}

Verificou-se que as primeiras pesquisas aplicadas com seres humanos na Análise do Comportamento foram realizadas no final da década de 50 e início da década de 60 , com os trabalhos realizados no Behavior Research Laboratory, por Lindsley, Skinner e Solomon, com pacientes psiquiátricos, e que as décadas de maior produção sobre o tema foram as de 60 e 70 . Como pôde ser verificado pela análise dos objetivos e dos comportamentos-alvo, inicialmente, objetivava-se apenas verificar a efetividade dos princípios operantes em indivíduos diagnosticados com esquizofrenia e não tratar os pacientes.

Todavia, a entrada dos analistas do comportamento nos hospitais psiquiátricos e a utilização de pacientes psicóticos como participantes de pesquisa, diferenciou a abordagem das demais correntes psicológicas existentes na época, que consideravam os comportamentos apresentados por essa população como sem solução.

As pesquisas mostraram que os pacientes psiquiátricos são sensíveis ao ambiente e que seus comportamentos são modificados pela aplicação de princípios operantes, possibilitando a intervenção em comportamentos tidos como indesejados. No entanto, as intervenções realizadas pelos chamados modificadores do comportamento não eram precedidas por análises de contingências e desconsideravam fatores importantes na manutenção dos comportamentos ditos problemas, alterando-os através da criação de contingências arbitrárias, que eram efetivas, mas não mantinham seus resultados a longo prazo e/ou na sua ausência.

As diversas críticas contribuíram para que os analistas do comportamento repensassem sua prática. A necessidade de realizar uma análise funcional (que é estritamente individual) dos comportamentos apresentados pelos pacientes psiquiátricos dificultou a realização de pesquisas com grupos, contribuindo para a retirada dos programas comportamentais dos hospitais (e talvez contribuindo para o atendimento restrito a consultórios).

Apesar das pesquisas mais recentes realizarem análise funcional (baseada no modelo de Iwata et al., 1994) do comportamento verbal dos pacientes psicóticos, principal "sintoma” dessa população, os resultados e a intervenção são muito semelhantes às realizadas anteriormente, quando as vocalizações inapropriadas eram vistas apenas como comportamentos a serem eliminados, a despeito da análise de operantes verbais proposta por Skinner.

Dessa forma, verifica-se que apesar das inquestionáveis descobertas na área, como a possibilidade de tratar pacientes psicóticos, uma vez que seus comportamentos são originados e mantidos por contingências ambientais, como os de qualquer outro ser humano, não foram realizadas novas pesquisas sobre o assunto nas últimas décadas. $\mathrm{O}$ cenário atual, pós reforma psiquiátrica, é diferente do encontrado nas primeiras pesquisas, nas quais os pacientes viviam em instituições fechadas (ambientes facilmente manipuláveis) e exige dos analistas do comportamento novas formas de atuação.

Ao contrário dos resultados encontrados na presente pesquisa sobre a produção de estudos estrangeiros enfocando o comportamento psicótico, observou-se que no Brasil há um aumento de produções de teses e dissertações sobre o tema nas últimas décadas. No entanto, na revisão realizada por Camargo (2008), que incluiu cinco periódicos brasileiros ${ }^{3}$, apenas dois artigos foram encontrados no período de 20 anos (1988 a 2007), o que indica que os registros de trabalhos são limitados, caso estejam sendo realizados estudos sobre o tema.

Sugere-se, portanto, a realização de uma revisão nos periódicos brasileiros, abrangendo o período posterior a 2007, a fim de verificar se há publicação dos estudos desenvolvidos e se apontam para novas possibilidades de intervenções junto a esta população.

3 Psicologia Teoria e Pesquisa, Psicologia Reflexão e Crítica, Psicologia Teoria e Prática, Revista Brasileira de Terapia Comportamental e Cognitiva e Revista Brasileira de Psiquiatria. 


\section{Referências}

American Psychiatric Association (2002). DSM-IVTR - Manual diagnóstico e estatístico de transtornos mentais. (Trad. D. Batista, $4^{\mathrm{a}}$. ed.) Porto Alegre: Artmed.

American Psychiatric Association (2013). Highlights of change from DSM-IV-TR to DSM-5. Retirado de http://www.dsm5.org/Documents/changes\%20from\%20dsm-iv-tr\%20to\%20dsm-5.pdf

Bach, P., \& Hayes, S. C. (2002). The use of Acceptance and Commitment Therapy to prevent the rehospitalization of psychotic patients: A randomized controlled trial. Journal of Consulting and Clinical Psychology, 7(5), 11291139. Retirado de http:/www.actmindfully.com. au/upimages/bach\%26hayes-_act_schizophrenia.pdf

Banaco, R. A., Zamignani, D. R. \& Meyer, S. B. (2010). Função do Comportamento e do DSM: terapeutas analítico-comportamentais discutem a psicopatologia. Em E. Z. Tourinho \& S. V. Luna. (Orgs.), Análise do Comportamento: investigações históricas, conceituais e aplicadas. (pp. 175-191). São Paulo: Roca.

Bandeira, M., Machado, E. L. \& Pereira, E. A. (2002). Reinserção Social de Psicóticos: componentes verbais e não-verbais do comportamento assertivo, em situações de fazer e receber críticas. Psicologia Reflexão e Crítica, 15(1), 89104. Retirado de http://www.scielo.br/pdf/prc/ v15n1/a11v15n1.pdf

Britto, I. A. G. S. (2005). Esquizofrenia: desafios para a ciência do comportamento. Em H. J. Guilhardi \& N. C. Aguirre. (Orgs.), Sobre Comportamento e Cognição. Expondo a variabilidade. (Vol. 16, pp. 38-44). Santo André, SP: Esetec.

Britto, I. A. G. S., Rodrigues, I. S., Alves, S. L. \& Quinta, T. L. S. S. (2010). Análise funcional de comportamentos verbais inapropriados de um esquizofrênico. Psicologia: Teoria e Pesquisa, 26(1), 139-144. Retirado de http://revistaptp. unb.br/index.php/ptp/article/viewArticle/307

Britto, I. A. G. S., Rodrigues, M. C. A., Santos, D. C. O. \& Ribeiro, M. A. (2006). Reforçamento diferencial de comportamentos verbais al- ternativos de um esquizofrênico. Revista Brasileira de Terapia Comportamental e Cognitiva, 8(1), 73-84. Retirado de http:// pepsic.bvsalud.org/sielo.php?pid=S1517$-55452006000100007 \& \mathrm{script}=\mathrm{sci}$ _art text\&tlng=en

Bueno, G. N. \& Britto, I. A. G. S. (2011). Uma abordagem funcional para os comportamentos de delirar e alucinar. Revista Brasileira de Terapia Comportamental e Cognitiva, 13(3), 4-15. Retirado de http://www.usp.br/rbtcc/index. php/RBTCC/article/view/458/338.

Camargo, M. I. C. P. (2008). O tratamento da esquizofrenia por analistas do comportamento: uma revisão de literatura. (Trabalho de Conclusão de Curso não publicado). Pontifícia Universidade Católica de São Paulo, São Paulo, SP.

Carmona, J. A., Cangas, A. J., García-Montes, J. M. \& Pedro, S. (2009). Terapia de Aceptación y Compromiso aplicada a los sintomas psicóticos. Em III Jornada Contextos. Retirado de http://www.uma.es/petra/contextos_nueva/ Jornadas3/Articulos/09_Carmona_y_

Montes_ACT_aplicada_a_los_sintomas_psicoticos.pdf

Dalgalarrondo, P. (2008). Psicopatologia e Semiologia dos transtornos mentais ( $2^{\mathrm{a}} \mathrm{ed}$.). Porto Alegre: Artmed.

Epaminondas, F. R. (2010). Modelagem de comportamento para controle de esquizofrenia (Dissertação de Mestrado não publicada). Pontifícia Universidade Católica de Goiás, Goiás, GO.

Felipe, G. R. (2009). Efeito das estratégias operantes em uma pessoa com o diagnóstico de esquizofrenia e família (Dissertação de Mestrado não publicada). Pontifícia Universidade Católica de Goiás, Goiás, GO.

García-Montes, J. M. \& Pérez-Álvarez, M. (2005). Fundamentación experimental y primeras aplicaciones clínicas de la Terapia de Aceptación y Compromiso (ACT) en el campo de los síntomas psicóticos. Revista Latinoamericana de Psicologia, 37(2), 379-393. Retirado de http:// pepsic.bvsalud.org/scielo.php?pid=S0120$-05342005000200010 \& \mathrm{script}=\mathrm{sci}$. arttext\&tlng=es 
García-Montes, J. M., Soriano, M. C. L., López, M. H., Basurto, F. Z. (2004). Aplicación de la Terapia de Aceptación y Compromiso (ACT) a sintomatología delirante: un estudio de caso. Psicothema, 6(1), 117-124. Retirado de http:// www.unioviedo.es/reunido/index.php/PST/article/view/8197/8061

Guedes, M. L. (1993). Equívocos da terapia comportamental. Temas em Psicologia, 2, 81-85. Retirado de http://pepsic. bvsalud.org/scielo.php?pid=S 1413 -389X1993000200011\&script=sci_arttext

Holland, J. G. (1978). Behaviorism: part of the problem or part of the solution? Journal of Applied Behavior Analysis, 11(1), 163-174. Retirado de http://www.ncbi.nlm.nih.gov/pmc/articles/ PMC1311279/

Hunziker, M. H. L. \& Samelo, M. J. (2011). Controle aversivo. Em N. B. Borges \& F. A. Cassas. Clínica Analítico-Comportamental: aspectos teóricos e práticos (pp. 49-63). Porto Alegre: Artmed.

Iwata, B. A., Dorsey, M. F., Slifer, K. J., Bauman, K. E., \& Richman, G. S. (1994). Toward a functional analysis of self-injury. Journal of Applied Behavior Analysis, 27(2), 197-209. Retirado de http://www.ncbi.nlm.nih.gov/pmc/articles/ pmc1297798/

Kazdin, A. E. (1982). The token economy: a decade later. Journal of Applied Behavior Analysis, 15(3), 431-445. Retirado de http://www.ncbi. nlm.nih.gov/pmc/articles/pmc1308287/

Kazdin, A. E., \& Bootzin, R. R. (1972). The token economy: an evaluation review. Journal of Applied Behavior Analysis, 5(3), 343-372. Retirado de http://www.ncbi.nlm.nih.gov/pmc/ articles/pmc1310772/

Lara, D. R., Gama, C. S., \& Abreu, P. S. B. (2004). Esquizofrenia. Em F. Kapczinski, J. Quevedo \& I. Izquierdo (Orgs.). Bases Biológicas dos Transtornos Psiquiátricos. (pp. 285-297). Porto Alegre: Artmed.

Layng, T. V. J., \& Andronis, P. T. (1984). Toward a functional analysis of Delusional Speech and hallucinatory behavior. The Behavior Analyst. 7(2), 139-156. Retirado de http://www.ncbi. nlm.nih.gov/pmc/articles/PMC2741750/
Louzã Neto, M. R., Azevedo, Y., \& Macedo, G. C. (2007). Esquizofrenia. Em C. N. Abreu. Síndromes psiquiátricas: diagnóstico e entrevista para profissionais de saúde mental. (pp. 55-63). Porto Alegre: Artmed.

Lundin, R. W. (1977). Personalidade: uma análise do comportamento (Trad. R. R. Kerbauy, $2^{\mathrm{a}}$. ed.). São Paulo: EPU. (Obra original publicada em 1969).

Maciel, A. T. B., \& Modesto, L. M. (2008, novembro). Esquizofrenia: Princípios Teóricos e Intervenção em Análise do Comportamento. Anais do II Encontro Paranaense de Análise do Comportamento, Paraná, PR, Brasil, 24.

Marcon, R. M. (2010). O comportamento verbal do esquizofrênico sob múltiplas condições de controle (Dissertação de Mestrado não publicada). Pontifícia Universidade Católica de Goiás, Goiás, GO.

Martone, M. C. C. (2005). Efeitos de contingências discriminativas sobre o repertório social e de higiene de uma participante institucionalizada (Dissertação de Mestrado não publicada). Pontifícia Universidade Católica de São Paulo, São Paulo, SP.

Martone, R. C., \& Zamignani, D. R. (2002). Esquizofrenia: A análise do comportamento tem o que dizer? Em H. J. Guilhard, M. B. B. P. Madi, P. P. Queiroz, \& M. C. Scoz (Orgs.), Sobre comportamento e cognição: contribuições para construção da teoria comportamental (Vol. 10, pp. 305-316). Santo André, SP: Esetec.

Miranda, E. (2005). A esquizofrenia sob a perspectiva dos princípios da análise do comportamento (Dissertação de Mestrado não publicada). Pontifícia Universidade Católica de Goiás, Goiás, GO.

Organização Mundial da Saúde (1993). Classificação Estatística Internacional de Doenças e Problemas Relacionados à Saúde (1 ${ }^{\text {a }}$. ed.). São Paulo: Edusp.

Pérez-Álvarez, M. (2004). Psychopathology According to Behaviorism: A Radical Restatement. The Spanish Journal of Psychology. 7(2), 171-177. Retirado de http://redalyc.uaemex.mx/redalyc/pdf/172/17207211.pdf 
Rutherford, A. (2003). Skinner boxes for psychotics: operant conditioning at Metropolitan State Hospital The Behavior Analyst. 26(2), 267-279. Retirado de http://www.ncbi.nlm. nih.gov/pmc/articles/PMC2731451/pdf/behavan00006-0089.pdf

Santana, L. A. M. (2008). Comportamento verbal e esquizofrenia: estratégia operante de intervenção (Dissertação de Mestrado não publicada). Pontifícia Universidade Católica de Goiás, Goiás, GO.

Santos, D. C. O. (2007). Análise da fala psicótica via estratégias operantes de intervenção (Dissertação de Mestrado não publicada). Pontifícia Universidade Católica de Goiás, Goiás, GO.

Scotti, J. R., McMorrow, M. J., \& Trawitzki, A. L. (1993). Behavioral treatment of chronic psychiatric disorders: publications trends and future directions. Behavioral Therapy, 24, 527550. Retirado de http://www.sciencedirect.com/ science/article/pii/S0005789405803168

Sidman, M. (2003). Coerção e suas implicações (Trad. M. A. Andery \& T. M. Sério). São Paulo: Livro Pleno. (Obra original publicada em 1989)

Silva, K. P. L. (2005). Análise aplicada e o comportamento diagnosticado como esquizofrênico (Dissertação de Mestrado não publicada). Pontifícia Universidade Católica de Goiás, Goiás, GO.

Silva, R. C. B. (2006). Esquizofrenia: uma revisão. Psicologia USP. 17(4), 263-285. Retirado de http:// revistasusp.sibi.usp.br/ scielo.php?script $=$ sci_arttext\&pid $=\mathrm{S} 1678$ $-1772006000400014 \& \operatorname{lng}=\mathrm{es} \& \mathrm{nrm}=\mathrm{isso}$

Skinner, B. F (1979). O que é comportamento psicótico? Em T. Millon (Org.), Teorias da Psicopatologia e Personalidade (pp. 188-196). Rio de Janeiro: Interamericana. (Obra original publicada em 1956)

Vilas Boas, D. L. O., Banaco, R. A., \& Borges, N. B. (2011). Discussões da análise do comportamento acerca dos transtornos psiquiátricos. Em N. B. Borges \& F. A. Cassas. Clínica AnalíticoComportamental: aspectos teóricos e práticos (pp. 95-101). Porto Alegre: Artmed.
Zanetti, M. V., \& Elkis, H. (2008). Esquizofrenia e outros transtornos psicóticos. Em P.G. Alvarenga \& A.G Andrade. Fundamentos em Psiquiatria (pp. 191-225). São Paulo; Manole.

\section{Anexo A}

\section{Referências dos artigos analisados}

Ayllon, T., \& Azrin, N. H. (1964). Reinforcement and instructions with mental patients. Journal of the Experimental Analysis of Behavior, 7, 327331. Retirado de http://www.ncbi.nlm.nih.gov/ pmc/articles/PMC1404247/

(1965). The measurement and reinforcement of behavior of psychotics. Journal of the Experimental Analysis of Behavior, 8, 357-383. Retirado de http://www. ncbi.nlm.nih.gov/pmc/articles/PMC1338117/

Ayllon, T., \& Haughton, E. (1962). Control of the behavior of schizophrenic patients by food. Journal of the Experimental Analysis of Behavior, 5, 343-352. Retirado de http://www.ncbi.nlm. nih.gov/pmc/articles/PMC1404104/

Ayllon, T., \& Michael, J. (1959). The psychiatric nurse as a behavioral engineer. Journal of the Experimental Analysis of Behavior, 2, 323-334. Retirado de http://www.ncbi.nlm.nih.gov/pmc/ articles/PMC1403907/

Bullock, D. H. (1959). Operant behavior in psychiatric patients. Journal of the Experimental Analysis of Behavior, 2, 262. Retirado de http://onlinelibrary.wiley.com/doi/10.1901/ jeab.1959.2-249/abstract

Dixon, M. R., Benedict, H., \& Larson, T. (2001). Functional analysis and treatment of inappropriate verbal behavior. Journal of Applied Behavior Analysis, 34, 361-363. Retirado de http://www.ncbi.nlm.nih.gov/pmc/articles/ PMC1284333/

Dunlap, G., Kern-Dunlap, L., Clarke, S., \& Robbins, F. R. (1991). Functional assessment, curricular revision, and severe behavior problems. Journal of Applied Behavior Analysis, 24, 387397. Retirado de http://www.ncbi.nlm.nih.gov/ pmc/articles/PMC1279582/ 
Fichter, M. M., Wallace, C. J., Liberman, R. P., \& Davis, J. R. (1976). Improving social interaction in a chronic psychotic using discrimination avoidance (nagging): Experimental analysis and generalization. Journal of Applied Behavior Analysis, 9, 377-386. Retirado de http://www. ncbi.nlm.nih.gov/pmc/articles/PMC1312034/

Fisher Jr, E. B. (1979). Overjustification effects in token economies. Journal of Applied Behavior Analysis, 12, 407-415. Retirado de http://www. ncbi.nlm.nih.gov/pmc/articles/PMC1311426/

Hersen, M., \& Bellack, A. S. (1976). A multiple-baseline analysis of social-skills training in chronic schizophrenics. Journal of Applied Behavior Analysis, 9, 239-245. Retirado de http://www. ncbi.nlm.nih.gov/pmc/articles/PMC1311938/

Borrero, J. C., Bartels-Meints , J. A., Sy, J. R., \& Francisco, M. T. (2011). Fixed-time schedule effects in combination with response-dependent schedules. Journal of Applied Behavior Analysis, 44, 163-167. Retirado de http://www. ncbi.nlm.nih.gov/pmc/articles/PMC3050458/

Kale, R. J., Kaye, J. H., Whelan, P. A., \& Hopkins, B. L. (1968). The effects of reinforcement on the modification, maintenance, and generalization of social responses of mental patients. Journal of Applied Behavior Analysis, 1, 307-314. Retirado de http://www.ncbi.nlm.nih.gov/pmc/articles/ PMC1311017/

Liberman, R. P., Teigen, J., Patterson, R., \& Baker, V. (1973). Reducing delusional speech in chronic paranoid schizophrenics. Journal of Applied Behavior Analysis, 6, 57-64. Retirado de http://www.ncbi.nlm.nih.gov/pmc/articles/ PMC1310806/

Mitchell, W. S., \& Stoffelmayr, B. E. (1973). Application of the Premack principle to the behavioral control of extremely inactive schizophrenics. Journal of Applied Behavior Analysis, 6, 419-423. Retirado de http://www.ncbi.nlm. nih.gov/pmc/articles/PMC1310854/

Nelson, G. L., \& Cone, J. D. (1979). Multiplebaseline analysis of a token economy for psychiatric inpatients. Journal of Applied Behavior Analysis, 12, 255-271. Retirado de http://www.ncbi.nlm.nih.gov/pmc/articles/ PMC1311367/
O’Brien, F., Azrin, N. H., \& Henson, K. (1969). Increasing communications of chronic mental patients by reinforcement and by response priming. Journal of Applied Behavior Analysis, 2, 23-29. Retirado de http://www.ncbi.nlm.nih. gov/pmc/articles/PMC1311031/

Patterson, R. L., \& Teigen, J. R. (1973). Conditioning and post-hospital generalization of nondelusional responses in a chronic psychotic patient. Journal of Applied Behavior Analysis, 6, 65-70. Retirado de http://www.ncbi.nlm.nih.gov/pub$\mathrm{med} / 16795396$

Ruskin, R. S., \& Maley, R. F. (1972). Item preference in a token economy ward store. Journal of Applied Behavior Analysis, 5, 373-378. Retirado de http://www.ncbi.nlm.nih.gov/pmc/articles/ PMC1310773/

Schraa, J. C., Lautmann, L., Luzi, M. K., \& Screven, C. G. (1978). Establishment of nondelusional responses in a socially withdrawn chronic schizophrenic. Journal of Applied Behavior Analysis, 11, 433-434. Retirado de http://www.ncbi.nlm. nih.gov/pmc/articles/PMC1311319/

Schraa, J. C., Lautmann, L., \& Screven, C. G. (1979). Increasing appropriate speech in a chronically schizophrenic. Journal of Applied Behavior Analysis, 12, 302. Retirado de http://www.ncbi. nlm.nih.gov/pmc/articles/PMC1311371/

Stewart, G., Van Houten, R., \& Van Houten, J. (1992). Increasing generalized social interactions in psychotic and mentally retarded residents through peer-mediated therapy. Journal of Applied Behavior Analysis, 25, 335-339. Retirado de http://www.ncbi.nlm.nih.gov/pmc/ articles/PMC1279714/

Wilder, D. A., Masuda, A., O'Connor, C., \& Baham, M. (2001). Brief functional analysis and treatment of bizarre vocalizations in an adult with schizophrenia. Journal of Applied Behavior Analysis, 34, 65-68. Retirado de http://www. ncbi.nlm.nih.gov/pmc/articles/PMC1284298/

Wincze, J. P., Leitenberg, H., \& Agras, W. S. (1972). The effects of token reinforcement and feedback on the delusional verbal behavior of chronic paranoid schizophrenics. Journal of Applied Behavior Analysis, 5, 247-262. Retirado de http://www.ncbi.nlm.nih.gov/pmc/articles/ PMC1310761/ 
Winkler, R. C. (1970). Management of chronic psychiatric patients by a token reinforcement system. Journal of Applied Behavior Analysis, 3, 47-55. Retirado de http://www.ncbi.nlm.nih. gov/pmc/articles/PMC1311089/

Wong, S. E., Terranova, M. D., Bowen, L., Zarate, R., Massel, H. K., \& Liberman, R. P. (1987). Providing independent recreational activities to reduce stereotypic vocalizations in chronic schizophrenics. Journal of Applied Behavior Analysis, 20, 77-81. Retirado de http://www. ncbi.nlm.nih.gov/pubmed/3583965

\section{Informações do Artigo}

Histórico do artigo:

Submetido em: 20/11/2013

Primeira decisão editorial: 06/08/2014

Segunda decisão editorial: 08/09/2014

Aceito para publicação em: 06/11/2014 